\title{
2D-DOA Estimation for Cylindrical Array with Mutual Coupling
}

\author{
Hao Feng, ${ }^{1,2}$ Lutao Liu, ${ }^{3}$ and Biyang Wen ${ }^{1}$ \\ ${ }^{1}$ School of Electronic Information, Wuhan University, Wuhan 430079, China \\ ${ }^{2}$ China Ship Development and Design Center, Wuhan 430000, China \\ ${ }^{3}$ College of Information and Telecommunication, Harbin Engineering University, Harbin 150001, China
}

Correspondence should be addressed to Lutao Liu; liulutao@msn.com

Received 14 January 2014; Accepted 15 February 2014; Published 30 March 2014

Academic Editor: Shen Yin

Copyright (C) 2014 Hao Feng et al. This is an open access article distributed under the Creative Commons Attribution License, which permits unrestricted use, distribution, and reproduction in any medium, provided the original work is properly cited.

\begin{abstract}
Most conventional direction-of-arrival (DOA) estimation algorithms are affected by the effect of mutual coupling, which make the performance of DOA estimation degrade. In this paper, a novel DOA estimation algorithm for conformal array in the presence of unknown mutual coupling is proposed. The special mutual coupling matrix (MCM) is applied to eliminate the effect of mutual coupling. With suitable array design, the decoupling between polarization parameter and angle information is accomplished. The two-demission DOA (2D-DOA) estimation is finally achieved based on estimation of signal parameters via rotational invariance techniques (ESPRIT). The proposed algorithm can be extended to conical conformal array as well. Two parameter pairing methods are illustrated for cylindrical and conical conformal array, respectively. The computer simulation verifies the effectiveness of the proposed algorithm.
\end{abstract}

\section{Introduction}

DOA estimation is a major part of array signal processing which is applied in various areas, such as smart antenna, wireless communication system, radar, sonar, and seismology [1-5]. The conventional algorithms such as the multiple signal classification (MUSIC) [6] and estimation of signal parameters via rotational invariance techniques (ESPRIT) [7] possess super-resolution ability. Recently, sparse signal representation and compressive sensing (CS) methods such as $l_{1}$-SVD [8] and JLZA-DOA [9] are applied to estimate DOA [10-16]. However, the DOA estimation performance will degrade severely due to the effect of mutual coupling.

In order to solve this problem, many algorithms have been proposed to deal with it. By adding instrumental elements, the reconstitution MCM can be used to eliminated the mutual coupling effect [17]. This method circumvented the difficulty of dealing with coherent signals in 2D-DOA estimations based on maximum likelihood (ML) method. However, the algorithm suffers from tremendous computational complexity. Conformal array is the array mounted on the curvature carrier [18]. It has many advantages such as reduction of aerodynamic drag, reduction of the weight of array, space saving, and reduction of radar cross-section (RCS) [19]. Several algorithms have been proposed for parameter estimation of conformal array recently. The joint polarization parameter and DOA estimation algorithm were proposed based on ESPRIT [20]. The four-order cumulant and ESPRIT were used for DOA estimation, which suffer from high computational complexity [21]. The subarray divided technique and MUSIC were adopted for DOA estimation for cylindrical conformal array $[22,23]$. The parallel factor (PARAFAC) analysis is used in [24] for 2D-DOA estimation. The space-time matrix and PARAFAC are used in [25] for joint frequency and 2D-DOA estimations. The state space and propagator method were used for joint frequency and 2DDOA estimations [26].

In this paper, a novel DOA estimation algorithm for conformal array in the presence of unknown mutual coupling is proposed. The selection matrix is applied to obtain the special MCM, which can eliminate the effect of mutual coupling. Then the decoupling between polarization parameter and angle information is accomplished with good array design. Two rotation invariance matrices are constructed for 2D-DOA estimation based on ESPRIT. Then the proposed algorithm is extended to conical conformal array. Two 
parameter pairing methods are illustrated for cylindrical conformal array, respectively. The proposed algorithm possesses good estimation performance according to the computer simulation results. Compared with ESPRIT algorithm, the computational complexity is not increasing, which is suitable for real-time application in cylindrical array system. In the future work, we will focus on the application of the proposed algorithm [27-29].

The organization of this paper is structured as follows. Section 2 introduces the snapshot data model for conformal array with unknown mutual coupling. Section 3 illustrates the mutual coupling elimination mechanism. Section 4 contains the core contributions of this paper, which give the DOA estimation algorithm in the presence of mutual coupling using ESPRIT. Section 5 presents the simulation results. Section 6 summarizes our conclusions.

\section{The Snapshot Data Model}

We construct the mathematic model of the conformal array using the model which is proposed in [21]. As shown in Figure 1(a), a narrowband far-field signal impinges on the conformal array. The elevation and azimuth are $\theta$ and $\varphi$, respectively. Thus the steering vector can be expressed as

$$
\begin{aligned}
\mathbf{a}(\theta, \varphi)= & {\left[h_{1} e^{-j 2 \pi\left(\left(\mathbf{p}_{1} \cdot \mathbf{u}\right) / \lambda\right)}, h_{2} e^{-j 2 \pi\left(\left(\mathbf{p}_{2} \cdot \mathbf{u}\right) / \lambda\right)},\right.} \\
& \left.\ldots, h_{2 M} e^{-j 2 \pi\left(\left(\mathbf{p}_{2 M} \cdot \mathbf{u}\right) / \lambda\right)}\right]^{T} \\
h_{m}= & \left(g_{m \theta}^{2}+g_{m \varphi}^{2}\right)^{1 / 2}\left(k_{\theta}^{2}+k_{\varphi}^{2}\right)^{1 / 2} \cos \left(\theta_{m g k}\right) \\
= & \left|\mathbf{g}_{m}\right|\left|\mathbf{p}_{l}\right| \cos \left(\theta_{m g k}\right) \\
= & \mathbf{g}_{m} \cdot \mathbf{p}_{l}=g_{m \theta} k_{\theta}+g_{m \varphi} k_{\varphi},
\end{aligned}
$$

where $\mathbf{p}_{i}(i=1,2, \ldots, 2 M)$ represents the distance vector between the $m$ th element and the origin point, $\mathbf{u}$ is the direction vector of the incident signal, and $\lambda$ is wavelength of the incident signal. As shown in Figure 1(b), $\mathbf{g}_{m}$ is the pattern of the $m$ th element and $g_{m \theta}$ and $g_{m \varphi}$ are the components of $\mathbf{g}_{m}$, which are projected onto two orthogonal directions of electric field of the incident signal, respectively. $k_{\theta}$ and $k_{\varphi}$ are polarization parameters. $h_{m}$ and $\mathbf{p}_{l}$ denote the $m$ th element's response to the incident signal and electric field vector, respectively. $\theta_{m g k}$ is the angle between $g_{m}$ and $\mathbf{p}_{l} \cdot(\cdot)^{T}$ denoting the transpose of matrix $(\cdot)$.

By substituting (2) into (1), we have

$$
\mathbf{a}(\theta, \varphi)=\mathbf{a}_{\theta}(\theta, \varphi)+\mathbf{a}_{\varphi}(\theta, \varphi),
$$

where

$$
\begin{gathered}
\mathbf{a}_{\theta}(\theta, \varphi)=\left[g_{1 \theta} k_{\theta} e^{-j 2 \pi\left(\left(\mathbf{p}_{1} \cdot \mathbf{u}\right) / \lambda\right)}, g_{2 \theta} k_{\theta} e^{-j 2 \pi\left(\left(\mathbf{p}_{2} \cdot \mathbf{u}\right) / \lambda\right)},\right. \\
\left.\ldots, g_{2 M \theta} k_{\theta} e^{-j 2 \pi\left(\left(\mathbf{p}_{2 M} \cdot \mathbf{u}\right) / \lambda\right)}\right]^{T}, \\
\mathbf{a}_{\varphi}(\theta, \varphi)=\left[g_{1 \varphi} k_{\varphi} e^{-j 2 \pi\left(\left(\mathbf{p}_{1} \cdot \mathbf{u}\right) / \lambda\right)}, g_{2 \varphi} k_{\varphi} e^{-j 2 \pi\left(\left(\mathbf{p}_{2} \cdot \mathbf{u}\right) / \lambda\right)},\right. \\
\left.\ldots, g_{2 M \varphi} k_{\varphi} e^{-j 2 \pi\left(\left(\mathbf{p}_{2 M} \cdot \mathbf{u}\right) / \lambda\right)}\right]^{T} .
\end{gathered}
$$

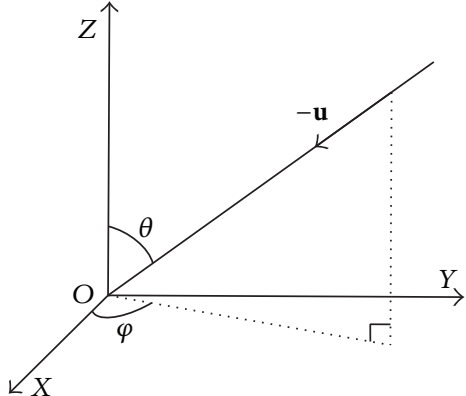

(a)

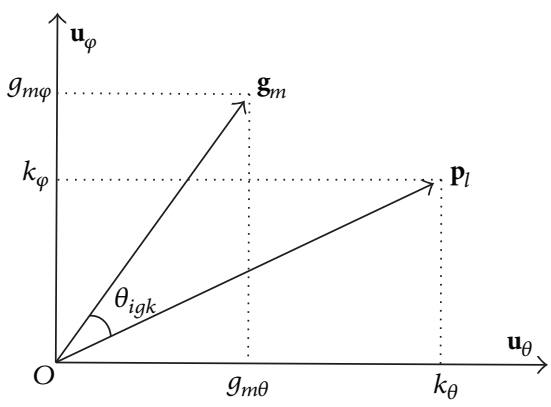

(b)

Figure 1: (a) The incident signal $\mathbf{u}$ and (b) the $m$ th sensors response.

Assume that $P$ uncorrelated narrowband plane waves with unknown DOAs impinge on the conformal array; the array output can be represented as

$$
\begin{aligned}
\mathbf{X}(n) & =\mathbf{G} \odot \mathbf{C A S}(n)+\mathbf{W}(n) \\
& =\mathbf{C}\left(\mathbf{G}_{\theta} \odot \mathbf{A}_{\theta} \mathbf{K}_{\theta}+\mathbf{G}_{\varphi} \odot \mathbf{A}_{\varphi} \mathbf{K}_{\varphi}\right) \mathbf{S}(n)+\mathbf{W}(n) \\
& =\mathbf{C B S}(n)+\mathbf{W}(n), \\
\mathbf{G}_{\theta}= & {\left[\mathbf{g}_{\theta}\left(\theta_{1}, \varphi_{1}\right), \mathbf{g}_{\theta}\left(\theta_{2}, \varphi_{2}\right), \ldots, \mathbf{g}_{\theta}\left(\theta_{P}, \varphi_{P}\right)\right], } \\
\mathbf{G}_{\varphi}= & {\left[\mathbf{g}_{\varphi}\left(\theta_{1}, \varphi_{1}\right), \mathbf{g}_{\varphi}\left(\theta_{2}, \varphi_{2}\right), \ldots, \mathbf{g}_{\varphi}\left(\theta_{P}, \varphi_{P}\right)\right], } \\
\mathbf{A}_{\theta}= & {\left[\mathbf{a}_{\theta}\left(\theta_{1}, \varphi_{1}\right), \mathbf{a}_{\theta}\left(\theta_{2}, \varphi_{2}\right), \ldots, \mathbf{a}_{\theta}\left(\theta_{P}, \varphi_{P}\right)\right], } \\
\mathbf{A}_{\varphi}= & {\left[\mathbf{a}_{\varphi}\left(\theta_{1}, \varphi_{1}\right), \mathbf{a}_{\varphi}\left(\theta_{2}, \varphi_{2}\right), \ldots, \mathbf{a}_{\varphi}\left(\theta_{P}, \varphi_{P}\right)\right], } \\
\mathbf{K}_{\theta}= & \operatorname{diag}\left(k_{1 \theta}, k_{2 \theta}, \ldots, k_{P \theta}\right), \\
\mathbf{K}_{\varphi}= & \operatorname{diag}\left(k_{1 \varphi}, k_{2 \varphi}, \ldots, k_{P \varphi}\right), \\
\mathbf{S}(n)= & {\left[s_{1}(n), s_{2}(n) \ldots, s_{P}(n)\right]^{T}, } \\
\mathbf{W}(n)= & {\left[w_{1}(n), w_{2}(n) \ldots, w_{2 M}(n)\right]^{T}, }
\end{aligned}
$$

where $\mathbf{G}$ and $\mathbf{A}$ denote the pattern matrix and the manifold matrix, respectively. “๑” stands for the Hadamard product. $\mathbf{K}_{\theta}$ and $\mathbf{K}_{\varphi}$ are the diagonal matrices whose diagonal entries are $k_{1 \theta}, k_{2 \theta}, \ldots, k_{P \theta}$ and $k_{1 \varphi}, k_{2 \varphi}, \ldots, k_{P \varphi}$, respectively. $k_{p \theta}$ and $k_{p \varphi}$ are polarization parameters of the $p$ th signal. $\mathbf{S}(n)$ denotes 
the signal vector. $\mathbf{W}(n)$ is additive Gaussian white noise $(\mathrm{AGWN})$ with zero mean and the covariance matrix is

$$
E\left\{\mathbf{W}(n) \mathbf{W}(n)^{H}\right\}=\mathbf{Q}=\sigma^{2} \mathbf{I}
$$

$(\cdot)^{H}$ denotes conjugate transpose of matrix $(\cdot) . \mathbf{I}$ is the identical matrix.

The matrix $\mathbf{C}$ is the MCM of the conformal array. Generally, the effect of mutual coupling among elements is ineluctable in practice. According to the reciprocity principle, the MCM is a symmetrical matrix.

Consider a scenario in which $N$ snapshots are accumulated; we have

$$
\mathbf{X}=\mathbf{C B S}+\mathbf{W}
$$

where $\mathbf{S}$ is the $P \times N$ signal matrix and $\mathbf{W}$ is the $2 M \times N$ noise matrix.

For ULA, the mutual coupling between adjacent elements is almost the same, and the magnitude of the coupling coefficients decays very fast along with the increasing element spacing. In essence, mutual coupling coefficients between two far apart elements can be approximated to zero. For simplicity, only the mutual coupling among elements which belongs to the identical ULA is considered in this paper. The mutual coupling degree of freedom is assumed to be $(q+1)$, which means that for the $i$ th element, the coupling comes from the $(i-q)$ th to $(i+q)$ th elements. Thus, the MCM C is modeled as a banded symmetric Toeplitz matrix, whose first row is $\mathbf{c}=\left[1, c_{1}, c_{2}, \ldots, c_{q}, 0, \ldots, 0\right]$ satisfying $0<\left|c_{q}\right|<$ $\left|c_{q-1}\right|<\cdots<c_{1}<1$. Then, MCM $\mathbf{C}$ is represented as

$$
\begin{aligned}
\mathbf{C} & =\operatorname{Toeplitz}(\mathbf{c}) \\
& =\operatorname{Toeplitz}\left\{\left[1, c_{1}, c_{2}, \ldots, c_{q}, 0, \ldots, 0\right]\right\} \\
& =\left[\begin{array}{cccccc}
1 & c_{1} & \cdots & c_{q} & \cdots & 0 \\
c_{1} & 1 & c_{1} & \cdots & \ddots & 0 \\
\vdots & c_{1} & 1 & \ddots & \cdots & c_{q} \\
c_{q} & \ldots & \ddots & \ddots & c_{1} & \vdots \\
0 & \ddots & \cdots & c_{1} & 1 & c_{1} \\
0 & \cdots & c_{q} & \cdots & c_{1} & 1
\end{array}\right] .
\end{aligned}
$$

Due to the varying curvature of the carrier, the pattern of each element is defined in the local coordinate, which means that the transformation from the global coordinate to the local coordinate should be done [25]. For the presence of the unknown mutual coupling, we must inhibit its effect. Furthermore, the polarization parameters couple with the signal parameters is another problem that we need to deal with.

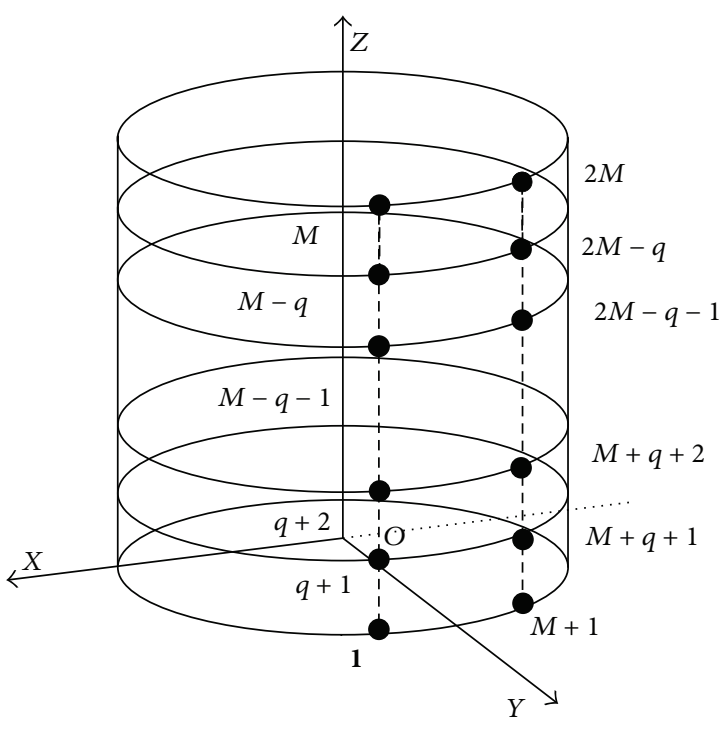

FIGURE 2: The structure of cylindrical conformal array.

\section{The Elimination of Effect of Unknown Mutual Coupling}

As shown in Figure 2, the neighboring elements spacing mounted on the same intersecting surface of the cylinder is $\lambda / 2$. The two adjacent intersecting surfaces spacing are $d=$ $\lambda / 2$. The radius of the cylinder is $5 \lambda$.

Based on the snapshot data model constructed above, we will give an elimination method of mutual coupling using the instrumental elements. In order to illustrate the mechanism how the effect of mutual coupling is eliminated, $1 \sim M$ elements are adopted as an example. The coordinate of each element is defined as $\left(x_{m}, y_{m}, z_{m}\right), m=1,2, \ldots, M . \mathbf{p}_{m}$ represents the position vector of the $m$ th element. $\vec{e}_{x}, \vec{e}_{y}$, and $\vec{e}_{z}$ represent the unit vector of $X$-axis, $Y$-axis, and $Z$-axis, respectively. $\mathbf{p}_{m}$ can be expressed as

$$
\mathbf{p}_{m}=x_{m} \vec{e}_{x}+y_{m} \vec{e}_{y}+z_{m} \vec{e}_{z}
$$

Because the elements are arranged on the same generatrix, we have $x_{1}=x_{2}=\cdots=x_{M}=x$ and $y_{1}=y_{2}=\cdots=$ $y_{M}=y$, respectively. Also, the elements possess the identical element's response $h_{1}$. The manifold matrix can be expressed as $\mathbf{B}_{1}=\left[\mathbf{b}_{1}\left(\theta_{1}\right), \mathbf{b}_{1}\left(\theta_{2}\right), \ldots, \mathbf{b}_{1}\left(\theta_{P}\right)\right]$, and the steering vector is

$$
\mathbf{b}_{1}\left(\theta_{p}\right)=\left[H_{p}, H_{p} v_{p}^{1}, \ldots, H_{p} v_{p}^{M-1}\right]^{T},
$$

where $v_{p}=\exp \left(-j 2 \pi d \cos \left(\theta_{p}\right) / \lambda\right)$ and

$$
H_{p}=h_{1} e^{-j 2 \pi\left(x \sin \left(\theta_{p}\right) \cos \left(\varphi_{p}\right)+y \sin \left(\theta_{p}\right) \sin \left(\varphi_{p}\right)\right) / \lambda} .
$$

In order to eliminate the effect of mutual coupling, the first and last $q$ elements of the $\bar{M}=(M-2 q)$-elements ULA are selected as instrumental elements [17]. At the first glance, it is an unreasonable idea to abandon some information that the array receives. However, the effect of mutual coupling is eliminated by this sacrifice. Define a selection matrix $\mathbf{F}=$ 
$\left[0_{(M-2 q) \times q}, \mathbf{I}_{M-2 q}, 0_{(M-2 q) \times q}\right]$; the output of the center array $\overline{\mathbf{X}}$ is expressed as [17]

$$
\overline{\mathbf{X}}=\mathrm{FCB}_{1} \mathrm{~S}+\mathrm{FW}=\overline{\mathbf{C}} \mathbf{B}_{1} \mathrm{~S}+\mathrm{FW}
$$

where the $\bar{M} \times M$ MCM $\overline{\mathbf{C}}$ of the center array is given by

$$
\overline{\mathbf{C}}=\mathbf{F C}
$$

$$
=\left[\begin{array}{cccccccc}
c_{q} & \cdots & 1 & \cdots & c_{q} & 0 & \cdots & 0 \\
0 & c_{q} & \cdots & 1 & \cdots & c_{q} & \cdots & 0 \\
\vdots & \ddots & \ddots & \cdots & \ddots & \cdots & \ddots & \vdots \\
0 & \cdots & 0 & c_{q} & \cdots & 1 & \cdots & c_{q}
\end{array}\right] .
$$

After adding the instrumental elements, an important relationship between MCM and the steering vector is represented as

$$
\begin{gathered}
\overline{\mathbf{C}}_{1}\left(\theta_{p}\right)=\left[\begin{array}{c}
H_{p}\left(c_{q}+\cdots+c_{1} v_{p}^{q-1}+v_{p}^{q}+c_{1} v_{p}^{q+1}+\cdots+c_{q} v_{p}^{2 q}\right) \\
H_{p}\left(c_{q} v_{p}+\cdots+c_{1} v_{p}^{q}+v_{p}^{q+1}+c_{1} v_{p}^{q+2}+\cdots+c_{q} v_{p}^{2 q+1}\right) \\
\vdots \\
H_{p}\left(c_{q} v_{p}^{M-2 q-2}+\cdots+c_{1} v_{p}^{M-q-2}+v_{p}^{M-q-1}+c_{1} v_{p}^{M-q}+\cdots+c_{q} v_{p}^{M-2}\right) \\
H_{p}\left(c_{q} v_{p}^{M-2 q-1}+\cdots+c_{1} v_{p}^{M-q-1}+v_{p}^{M-q}+c_{1} v_{p}^{M-q+1}+\cdots+c_{q} v_{p}^{M-1}\right)
\end{array}\right] \\
=H_{p}\left(c_{q}+\cdots+c_{1} v_{p}^{q-1}+v_{p}^{q}+c_{1} v_{p}^{q+1}+\cdots+c_{q} v_{p}^{2 q}\right)\left[\begin{array}{c}
1 \\
v_{p} \\
\vdots \\
v_{p}^{M-1}
\end{array}\right] \\
=H_{p} v_{p}^{q}\left(2 \sum_{q^{\prime}=1}^{q} c_{q^{\prime}} \cos \left(\frac{2 q^{\prime} \pi \sin \left(\theta_{p}\right) d}{\lambda}\right)+1\right) \overline{\mathbf{b}}_{1}\left(\theta_{p}\right) \\
=c\left(\theta_{p}\right) \overline{\mathbf{b}}_{1}\left(\theta_{p}\right),
\end{gathered}
$$

where $\overline{\mathbf{b}}_{1}\left(\theta_{p}\right)$ is the ideal steering vector of the center array $\left(q+1 \sim M-q\right.$ elements) and $c\left(\theta_{p}\right)$ is a scalar only concerned with the mutual coupling coefficients and the direction of the incident signal $\theta_{p} \cdot c\left(\theta_{p}\right)$ is defined as

$$
c\left(\theta_{p}\right)=H_{p} v_{p}^{q}\left(2 \sum_{q^{\prime}=1}^{q} c_{q^{\prime}} \cos \left(\frac{2 q^{\prime} \pi \sin \left(\theta_{p}\right) d}{\lambda}\right)+1\right) .
$$

Thus, the covariance matrix of the centre array is given by

$$
\begin{aligned}
\overline{\mathbf{R}}_{x} & =E\left\{\overline{\mathbf{X}} \overline{\mathbf{X}}^{H}\right\}=\overline{\mathbf{C}} \mathbf{B}_{1} \overline{\mathbf{R}}_{s} \mathbf{B}_{1}^{H} \overline{\mathbf{C}}^{H}+\sigma^{2} \mathbf{I} \\
& =\overline{\mathbf{B}}_{1} \mathbf{D} \overline{\mathbf{R}}_{s} \mathbf{D}^{H} \overline{\mathbf{B}}_{1}^{H}+\sigma^{2} \mathbf{I},
\end{aligned}
$$

where

$$
\begin{aligned}
& \overline{\mathbf{B}}_{1}=\left[\overline{\mathbf{b}}_{1}\left(\theta_{1}\right), \overline{\mathbf{b}}_{1}\left(\theta_{2}\right), \ldots, \overline{\mathbf{b}}_{1}\left(\theta_{P}\right)\right], \\
& \mathbf{D}=\operatorname{diag}\left\{c\left(\theta_{1}\right), c\left(\theta_{2}\right), \ldots, c\left(\theta_{P}\right)\right\} .
\end{aligned}
$$

Assume that the power of the $p$ th incident signal is $\sigma_{p}^{2}$. Equation (16) can be written as

$$
\overline{\mathbf{R}}_{x}=\overline{\mathbf{B}}_{1} \mathbf{R} \overline{\mathbf{B}}_{1}^{H}+\sigma^{2} \mathbf{I},
$$

where

$$
\mathbf{R}=\operatorname{diag}\left\{\sigma_{1}^{2}\left|c\left(\theta_{1}\right)\right|^{2}, \sigma_{2}^{2}\left|c\left(\theta_{2}\right)\right|^{2}, \ldots, \sigma_{P}^{2}\left|c\left(\theta_{P}\right)\right|^{2}\right\} .
$$

\section{DOA Estimation Based On ESPRIT}

We can deal with the coupling between the polarization parameters and the signal parameters after the mutual coupling is eliminated. Define two $(\bar{M}-1) \times \bar{M}$

$$
\mathbf{P}_{1}=\left[\begin{array}{cccc}
1 & \cdots & 0 & 0 \\
\vdots & \ddots & \vdots & \vdots \\
0 & \cdots & 1 & 0
\end{array}\right], \quad \mathbf{P}_{2}=\left[\begin{array}{cccc}
0 & 1 & \cdots & 0 \\
\vdots & \vdots & \ddots & \vdots \\
0 & 0 & \cdots & 0
\end{array}\right] .
$$

Choosing $q+1 \sim M-q-1, q+2 \sim M-q, q+1 \sim M-q$, and $M+q+1 \sim 2 M-q$ elements constitutes array 1 , array 2 , array 3 , and array 4 , respectively. The distance vector between array 1 and 2 is $\Delta \mathbf{P}_{1}$ and $d_{1}=\left|\Delta \mathbf{P}_{1}\right|=\lambda / 2$. The distance vector between arrays 1 and 3 is $\Delta \mathbf{P}_{2}$, which is shown in Figure 3 . The elements' patterns of the same generatrix are identical. With special array design, both the eliminations of effect of mutual coupling and the decoupling between polarization parameter and angle information can be realized.

Figures 2 and 3 show the array structure. In the global coordinate, the elevation and azimuth of $\Delta \mathbf{P}_{1}$ and $\Delta \mathbf{P}_{2}$ can be 


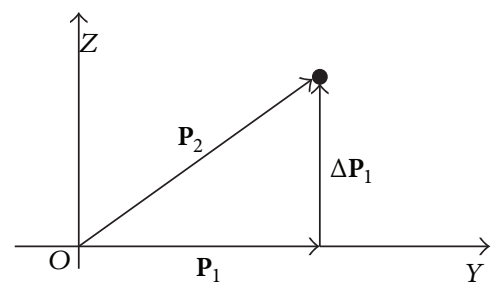

(a) The distance vector $\Delta \mathbf{P}_{1}$

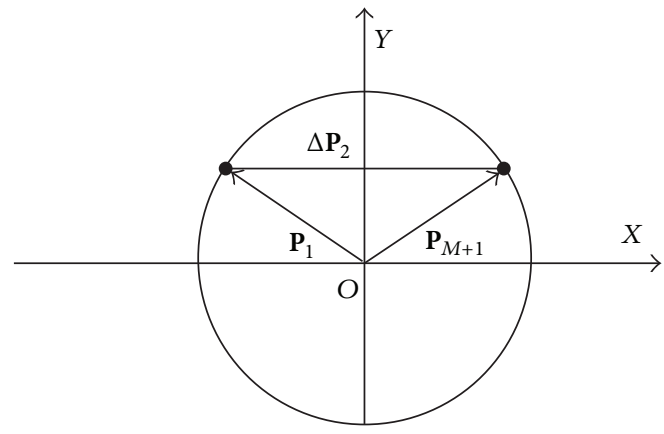

(b) The distance vector $\Delta \mathbf{P}_{2}$

FIgure 3: The distance vector.

represented as $\theta_{\Delta \mathbf{P}_{1}}=0, \varphi_{\Delta \mathbf{P}_{1}}=\pi / 2$, and $\theta_{\Delta \mathbf{P}_{2}}=\pi / 2, \varphi_{\Delta \mathbf{P}_{2}}=$ 0 , respectively.

Define a novel array output vector $\overline{\mathbf{Z}}_{1}=\left[\overline{\mathbf{X}}_{1}^{T}, \overline{\mathbf{X}}_{2}^{T}\right]^{T}$; the covariance matrix is represented as

$$
\begin{aligned}
& \overline{\mathbf{R}}_{z 1}=\left[\begin{array}{ll}
E\left\{\overline{\mathbf{X}}_{1} \overline{\mathbf{X}}_{1}^{H}\right\} & E\left\{\overline{\mathbf{X}}_{1} \overline{\mathbf{X}}_{2}^{H}\right\} \\
E\left\{\overline{\mathbf{X}}_{2} \overline{\mathbf{X}}_{1}^{H}\right\} & E\left\{\overline{\mathbf{X}}_{2} \overline{\mathbf{X}}_{2}^{H}\right\}
\end{array}\right] \\
& =\left[\begin{array}{lll}
\mathbf{P}_{1} \overline{\mathbf{R}}_{x} \mathbf{P}_{1}^{T} & \mathbf{P}_{1} \overline{\mathbf{R}}_{x} \mathbf{P}_{2}^{T} \\
\mathbf{P}_{2} \overline{\mathbf{R}}_{x} \mathbf{P}_{1}^{T} & \mathbf{P}_{2} \overline{\mathbf{R}}_{x} \mathbf{P}_{2}^{T}
\end{array}\right] \\
& =\left[\begin{array}{c}
\overline{\mathbf{B}}_{11} \\
\overline{\mathbf{B}}_{11} \boldsymbol{\Psi}_{1}
\end{array}\right] \mathbf{R}\left[\begin{array}{c}
\overline{\mathbf{B}}_{11} \\
\overline{\mathbf{B}}_{11} \boldsymbol{\Psi}_{1}
\end{array}\right]^{H}+\sigma_{n}^{2} \boldsymbol{\Sigma},
\end{aligned}
$$

where $\overline{\mathbf{B}}_{11}=\mathbf{P}_{1} \overline{\mathbf{B}}_{1}$ and $\boldsymbol{\Psi}_{1}$ are expressed as

$$
\begin{gathered}
\boldsymbol{\Psi}_{1}=\operatorname{diag}\left[\exp \left(-j \omega_{11}\right), \exp \left(-j \omega_{12}\right), \ldots, \exp \left(-j \omega_{1 P}\right)\right] \\
\begin{aligned}
& \omega_{1 p}=\left(\frac{2 \pi}{\lambda}\right) \Delta \mathbf{P}_{1} \cdot \mathbf{u}_{p} \\
&=\left(\frac{2 \pi d_{1}}{\lambda}\right)[ \sin \left(\theta_{\Delta \mathbf{P}_{1}}\right) \cos \left(\varphi_{\Delta \mathbf{P}_{1}}\right) \sin \left(\theta_{p}\right) \cos \left(\varphi_{p}\right) \\
&+\sin \left(\theta_{\Delta \mathbf{P}_{1}}\right) \sin \left(\varphi_{\Delta \mathbf{P}_{1}}\right) \sin \left(\theta_{p}\right) \sin \left(\varphi_{p}\right) \\
&\left.+\cos \left(\theta_{\Delta \mathbf{P}_{1}}\right) \cos \left(\theta_{p}\right)\right] .
\end{aligned}
\end{gathered}
$$

Regardless of the noise $\sigma_{n}^{2} \boldsymbol{\Sigma}$ the signal subspace $\overline{\mathbf{E}}_{S 1}$ is obtained by taking the eigenvalue decomposition (EVD) of
$\overline{\mathbf{R}}_{z}$. Then $\overline{\mathbf{E}}_{S 1}$ is divided into two submatrices (the top and the bottom), and we have a full rank matrix $\mathbf{T}$ satisfying

$$
\overline{\mathbf{E}}_{S 1}=\left[\begin{array}{l}
\overline{\mathbf{E}}_{S 11} \\
\overline{\mathbf{E}}_{S 12}
\end{array}\right]=\left[\begin{array}{c}
\overline{\mathbf{B}}_{11} \mathbf{T} \\
\overline{\mathbf{B}}_{11} \boldsymbol{\Psi}_{1} \mathbf{T}
\end{array}\right] .
$$

We can estimate $\Phi_{1}$ by

$$
\begin{gathered}
\boldsymbol{\Phi}_{1}=\left(\overline{\mathbf{E}}_{S 11}^{H} \overline{\mathbf{E}}_{S 11}^{H}\right)^{-1} \overline{\mathbf{E}}_{S 11}^{H} \overline{\mathbf{E}}_{S 12}, \\
\boldsymbol{\Psi}_{1}=\mathbf{T} \boldsymbol{\Phi}_{1} \mathbf{T}^{-1} .
\end{gathered}
$$

The diagonal matrix constructed by the eigenvalue $t_{1 p}$ of $\Phi_{1}$ must be equal to $\Psi_{1}$. The columns of matrix $\mathbf{T}$ construct the eigenvectors of $\Psi_{1}$. Then array 3 and array 4 can construct a group array. Thus the output of the centre array 3 and array 4 is given by

$$
\begin{aligned}
& \overline{\mathbf{X}}_{3}=\overline{\mathbf{C}}_{1} \mathbf{B}_{2} \mathbf{S}+\sigma^{2} \mathbf{I}, \\
& \overline{\mathbf{X}}_{4}=\overline{\mathbf{C}}_{1} \mathbf{B}_{2} \boldsymbol{\Psi}_{2} \mathbf{S}+\sigma^{2} \mathbf{I},
\end{aligned}
$$

respectively, where $\overline{\mathbf{C}}_{1}$ represents the $(M-2 p) \times(M-2 p)$ MCM and $\mathbf{B}_{2}$ is the $(M-2 p) \times P$ manifold matrix of array. The group array output is defined as $\overline{\mathbf{Z}}_{2}=\left[\overline{\mathbf{X}}_{3}^{T}, \overline{\mathbf{X}}_{4}^{T}\right]^{T}$.

The covariance matrix can be expressed as

$$
\begin{aligned}
& \overline{\mathbf{R}}_{z 2}=\left[\begin{array}{ll}
E\left\{\overline{\mathbf{X}}_{3} \overline{\mathbf{X}}_{3}^{H}\right\} & E\left\{\overline{\mathbf{X}}_{3} \overline{\mathbf{X}}_{4}^{H}\right\} \\
E\left\{\overline{\mathbf{X}}_{4} \overline{\mathbf{X}}_{3}^{H}\right\} & E\left\{\overline{\mathbf{X}}_{4} \overline{\mathbf{X}}_{4}^{H}\right\}
\end{array}\right] \\
& =\left[\begin{array}{c}
\overline{\mathbf{B}}_{2} \\
\overline{\mathbf{B}}_{2} \Psi_{2}
\end{array}\right] \mathbf{R}\left[\begin{array}{c}
\overline{\mathbf{B}}_{2} \\
\overline{\mathbf{B}}_{2} \boldsymbol{\Psi}_{2}
\end{array}\right]^{H}+\sigma_{n}^{2} \boldsymbol{\Sigma}_{1}, \\
& \Psi_{2}=\operatorname{diag}\left[\frac{h_{2}\left(\theta_{1}, \varphi_{1}\right)}{h_{1}\left(\theta_{1}, \varphi_{1}\right)} \exp \left(-j \omega_{21}\right), \frac{h_{2}\left(\theta_{2}, \varphi_{2}\right)}{h_{1}\left(\theta_{2}, \varphi_{2}\right)}\right. \\
& \left.\times \exp \left(-j \omega_{22}\right), \ldots, \frac{h_{2}\left(\theta_{P}, \varphi_{P}\right)}{h_{1}\left(\theta_{P}, \varphi_{P}\right)} \exp \left(-j \omega_{2 P}\right)\right], \\
& \omega_{2 p}=\left(\frac{2 \pi}{\lambda}\right) \Delta \mathbf{P}_{2} \cdot u_{i} \\
& =\left(\frac{2 \pi d_{2}}{\lambda}\right)\left[\sin \left(\theta_{\Delta \mathbf{P}_{2}}\right) \cos \left(\varphi_{\Delta \mathbf{P}_{2}}\right) \sin \left(\theta_{p}\right) \cos \left(\varphi_{p}\right)\right. \\
& +\sin \left(\theta_{\Delta \mathbf{P}_{2}}\right) \sin \left(\varphi_{\Delta \mathbf{P}_{2}}\right) \sin \left(\theta_{p}\right) \sin \left(\varphi_{p}\right) \\
& \left.+\cos \left(\theta_{\Delta \mathbf{P}_{2}}\right) \cos \left(\theta_{p}\right)\right] \text {, }
\end{aligned}
$$

where $\theta_{\Delta \mathbf{P}_{i}}$ and $\varphi_{\Delta \mathbf{P}_{i}}(i=1,2)$ represent the elevation and azimuth of the distance vector in the global coordinate, respectively.

The subspaces of array 3 and array 4 are defined as $\overline{\mathbf{E}}_{S 21}$ and $\overline{\mathbf{E}}_{S 22}$, respectively. $\boldsymbol{\Phi}_{2}$ is similarly defined as $\boldsymbol{\Phi}_{1}$, which can be solved by

$$
\boldsymbol{\Phi}_{2}=\left(\overline{\mathbf{E}}_{S 21}^{H} \overline{\mathbf{E}}_{S 21}^{H}\right)^{-1} \overline{\mathbf{E}}_{S 21}^{H} \overline{\mathbf{E}}_{S 22} .
$$


There exists an invertible matrix $\mathbf{T}$, satisfying

$$
\Psi_{2}=\mathbf{T}_{1} \Phi_{2} \mathbf{T}_{1}^{-1}
$$

where $\Psi_{2}$ can be obtained by taking the EVD of matrix $\boldsymbol{\Phi}_{2}$ and the eigenvalue of it is defined as $t_{2 p}$. There may exist phase ambiguous caused by the positive and negative of $h_{1}$ and $h_{2}$. Because $h_{1}$ and $h_{2}$ are real numbers, this problem can be solved by squaring the eigenvalue $t_{2 p}$ :

$$
\begin{gathered}
\omega_{2 p}=-\frac{1}{2} \angle\left(\left[\frac{h_{2}\left(\theta_{p}, \varphi_{p}\right)}{h_{1}\left(\theta_{p}, \varphi_{p}\right)} \exp \left(-j \omega_{2 p}\right)\right]^{2}\right) \\
=-\frac{1}{2} \angle\left(\exp \left(-j 2 \omega_{2 p}\right)\right)=-\frac{1}{2} \angle\left(\left(t_{2 p}\right)^{2}\right), \\
\omega_{1 p}=-\angle\left(\exp \left(-j \omega_{1 p}\right)\right)=-\angle\left(t_{1 p}\right),
\end{gathered}
$$

where $\angle$ is the angle of complex. Based on (25) and (31), the elevation $\theta_{p}$ and azimuth $\varphi_{p}$ can be obtained by

$$
\begin{gathered}
\theta_{p}=\arccos \left(\frac{\lambda t_{1 p}}{2 \pi d_{1}}\right)=\arccos \left(\frac{t_{1 p}}{\pi}\right), \\
\varphi_{p}=\arccos \left(\frac{\lambda t_{2 p}}{2 \pi d_{2} \sin \left(\theta_{p}\right)}\right)=\arccos \left(\frac{2 t_{2 p}}{\pi \sin \left(\theta_{p}\right)}\right) .
\end{gathered}
$$

Then the DOA estimation in the presence of mutual coupling is accomplished. However, when $P \geq 2$, the parameter pairing between $\omega_{1 i}(i=1,2, \ldots, P)$ and $\omega_{2 j}(i=$ $1,2, \ldots, P)$ is a big problem. The eigenvectors of the identical source are strongly correlated, and thus we can construct the sequencing matrix G. The parameter pairing can be accomplished by adjusting the order of eigenvectors.

$\mathbf{T}_{1}$ and $\mathbf{T}_{2}$ are eigenvectors which correspond to $\overline{\boldsymbol{\Psi}}_{1}$ and $\bar{\Psi}_{2}$, respectively. We have the equation as follows:

$$
\mathbf{G}_{1}=\mathbf{T}_{1}^{H} \mathbf{T}_{2}
$$

According to the coordinate of the maximal entry in the columns (or rows) of matrices G, we adjust the order of eigenvectors. Then the parameter pairing is completed.

\section{Simulation Results}

For the sake of simplicity, only the cylindrical conformal array is utilized to simulate the experiments as follows: the array structure is shown in Figure 2. The number of elements is 16; that is, $M=8$. The lowest order circular patch model $[21,30]$ is used in the simulation, and the element pattern is given by

$$
\begin{aligned}
g_{p \theta}(\theta, \varphi)= & \left\{J_{2}\left(\frac{\pi d}{\lambda} \sin \theta_{m}^{*}\right)-J_{0}\left(\frac{\pi d}{\lambda} \sin \theta_{m}^{*}\right)\right\} \\
& \times\left(\cos \varphi_{m}^{*}-j \sin \varphi_{m}^{*}\right), \quad 0 \leq \theta \leq \frac{\pi}{2}, \\
g_{p \varphi}(\theta, \varphi)= & \left\{J_{2}\left(\frac{\pi d}{\lambda} \sin \theta_{m}^{*}\right)+J_{0}\left(\frac{\pi d}{\lambda} \sin \theta_{m}^{*}\right)\right\}
\end{aligned}
$$

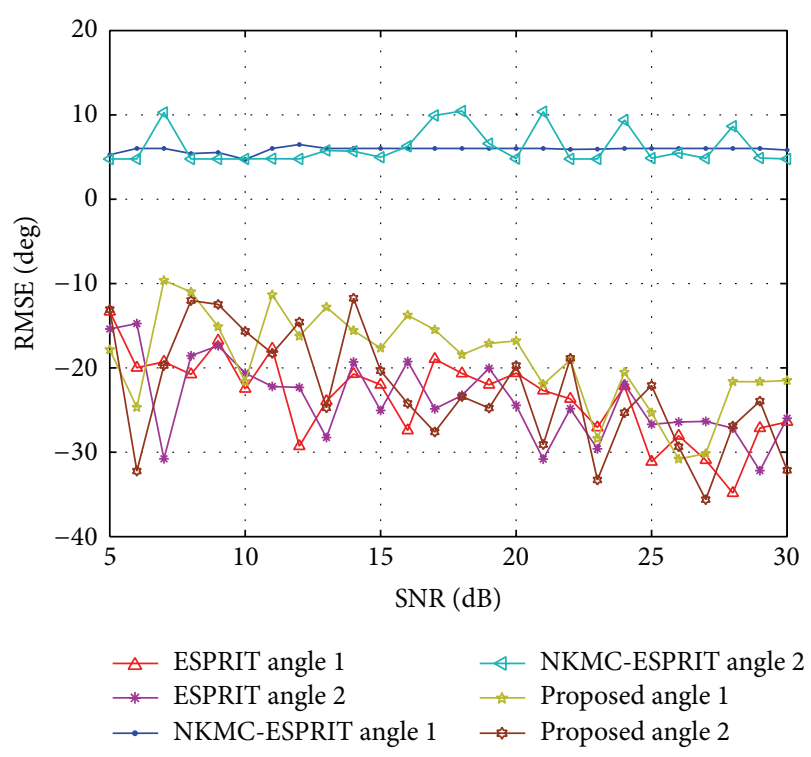

(a) The RMSE against SNR

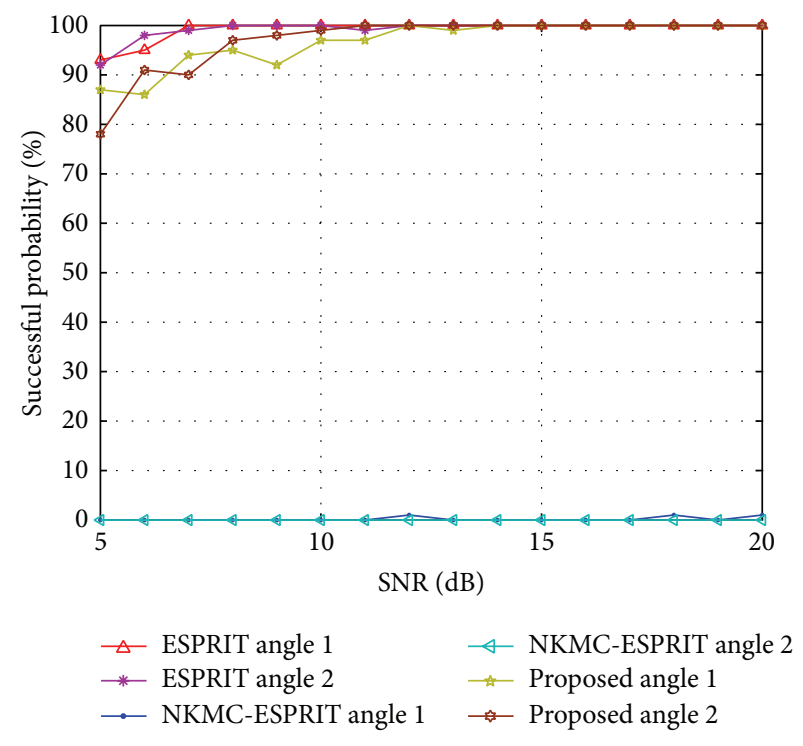

(b) The successful probability against SNR

FIGURE 4: The performance against SNR.

$$
\begin{gathered}
\times \cos \theta_{m}^{*}\left(\sin \varphi_{m}^{*}-j \cos \varphi_{m}^{*}\right), \quad 0 \leq \theta \leq \frac{\pi}{2}, \\
g_{p \theta}\left(\theta_{m}^{*}, \varphi_{m}^{*}\right)=g_{p \varphi}\left(\theta_{m}^{*}, \varphi_{m}^{*}\right)=0, \quad \text { otherwise, }
\end{gathered}
$$

where $\theta_{j}^{*}$ and $\varphi_{j}^{*}$ stand for the elevation and azimuth of the $p$ th incident signal in the $m$ th local coordinate, respectively.

The RMSE against SNR is plotted at first, which is shown in Figure 4(a). Two spatially uncorrelated narrowband incident signals impinge on the cylindrical conformal array with the elevation and azimuth of $\left(100^{\circ}, 60^{\circ}\right)$ and $\left(95^{\circ}, 50^{\circ}\right)$, respectively. The measurements are corrupted by spatially 
and temporally uncorrelated additive Gaussian white noise AGWN. Without loss of generality, $k_{1 \theta}=0.5, k_{1 \varphi}=0.5 ; k_{2 \theta}=$ $0.3, k_{2 \varphi}=0.7$. The number of mutual coupling coefficients is $q=2$, and $c_{1}=0.4500+i 0.5362, c_{2}=0.2598-i 0.1500$. The number of snapshots is 200. 100 Monte Carlo trials are simulated under the parameters above and the following experiments. It is shown in Figure 4(a) that RMSE of NKMCESPRIT is extremely larger than that of ESPRIT and the proposed algorithm. The unknown mutual coupling leads to the degradation of the estimation performance. In this scenario, NKMC-ESPRIT could not obtain the actual DOAs of the incident signals. The RMSE of the proposed algorithm is slightly larger than that of ESPRIT. This is caused by the aperture loss of the proposed algorithm; only 12 elements are applied to estimate DOA, which are less than that of ESPRIT (16 elements).

Figure 4(b) shows the successful probability against SNR of the three different algorithms. The successful probability of NKMC-ESPRIT approximates to zero, which implies that NKMC-ESPRIT could not estimate DOA accurately. The result is identical with the large RMSE of NKMC-ESPRIT mentioned above. The successful probability of ESPRIT and the proposed algorithm increase with the SNR increases. When SNR is larger than $12 \mathrm{~dB}$, the successful probability of ESPRIT is almost $100 \%$. The proposed algorithm needs SNR to be larger than $14 \mathrm{~dB}$. Without the effect of mutual coupling, ESPRIT outperforms the proposed algorithm. However, the proposed algorithm possesses good estimation performance with unknown mutual coupling.

The RMSE against snapshot number is shown in Figure 5(a). SNR is $0 \mathrm{~dB}$. Other simulation conditions are identical with the first simulation. It is shown in Figure 5(a) that RMSE of NKMC-ESPRIT is stable with the varying snapshot number. It means that NKMC-ESPRIT could not be used for DOA estimation because of the effect of mutual coupling. RMSE of proposed algorithm is slightly larger than that of ESPRIT. The aperture loss caused by the elimination mechanism of mutual coupling leads to this result. However, with snapshot number increases, RMSE of ESPRIT and the proposed algorithm are not varied too much. This result shows that RMSE is not affected by the varying snapshot number.

The successful probability against snapshot number is shown in Figure 5(b). The successful probability of NKMCESPRIT approximates to zero with varying snapshot number. When snapshot number is larger than 500, the successful probability of ESPRIT is almost $100 \%$. The proposed algorithm needs 1000 snapshot number to achieve $95 \%$ successful probability. The low SNR mainly leads to this large requirement of snapshot number. This simulation gives different result compared with RMSE simulation, which shows that the successful probability is improved with the snapshot number increases.

\section{Conclusion}

In this paper, a novel DOA estimation algorithm for conformal array in the presence of unknown mutual coupling is
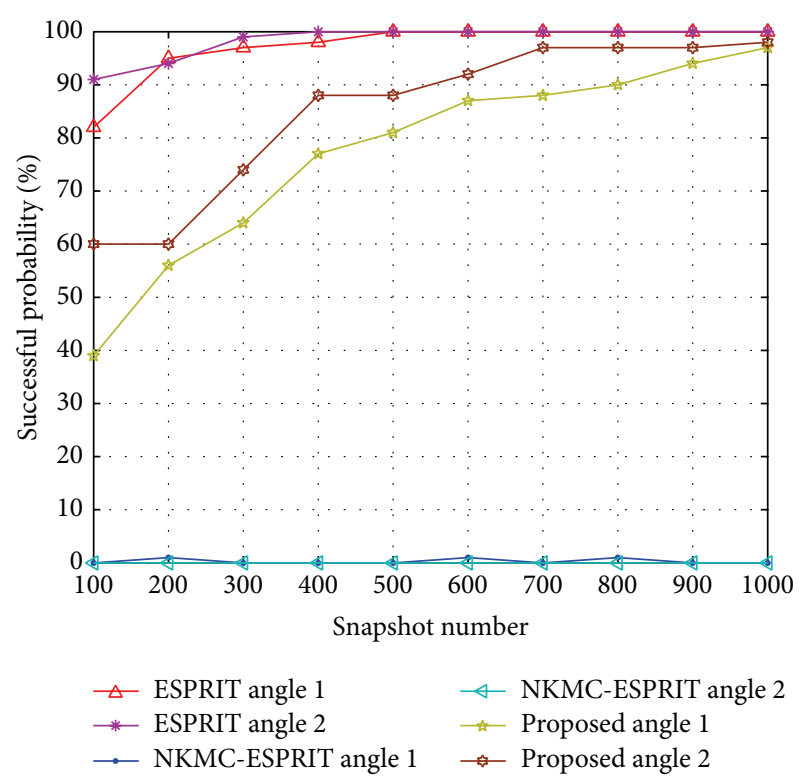

(a) The RMSE against snapshot number

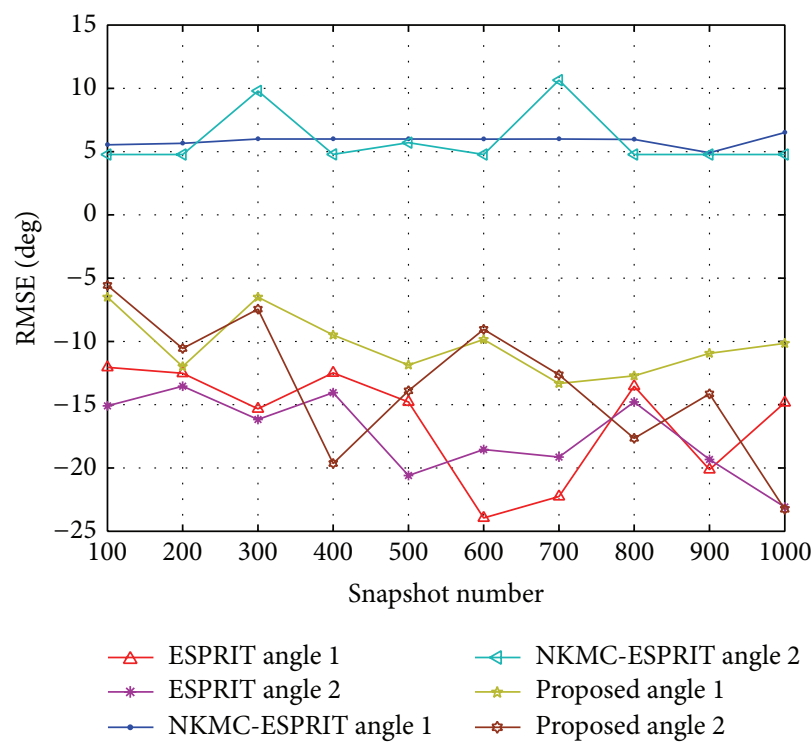

(b) The successful probability against snapshot number

FIGURE 5: The performance against snapshot number.

proposed. The special MCM is applied to eliminate the effect of mutual coupling. With good array design, the decoupling between polarization parameter and angle information is accomplished. The 2D-DOA estimation is finally achieved based on ESPRIT algorithm. The proposed algorithm can also be extended to conical conformal array. The computer simulation verifies the effectiveness of the proposed algorithm.

\section{Conflict of Interests}

The paper is approved by all authors for publication. The authors declare that there is no conflict of interests regarding the publication of this paper. 


\section{Disclosure}

One of the authors would like to declare on behalf of other coauthors that the work described is original research that has not been published previously.

\section{References}

[1] H. Krim and M. Viberg, "Two decades of array signal processing research: the parametric approach," IEEE Signal Processing Magazine, vol. 13, no. 4, pp. 67-94, 1996.

[2] D. H. Johnson and D. E. Dudgeon, Array Signal ProcessingConcepts and Techniques, Prentice Hall, Englewood Cliffs, NJ, USA, 1993.

[3] P. Stocia and R. Moses, Introduction to Spectral Analysis, Prentice Hall, Upper Saddle River, NJ, USA, 2nd edition, 2004.

[4] S. Yin, S. Ding, A. Haghani, H. Hao, and P. Zhang, "A comparison study of basic datadriven fault diagnosis and process monitoring methods on the benchmark Tennessee Eastman process," Journal of Process Control, vol. 22, no. 9, pp. 1567-1581, 2012.

[5] S. Yin, H. Luo, and S. Ding, "Real-time implementation of faulttolerant control systems with performance optimization," IEEE Transactions on Industrial Electronics, vol. 64, no. 5, pp. $2402-$ 2411, 2014.

[6] R. O. Schmidt, "Multiple emitter location and signal parameter estimation," IEEE Transactions on Antennas and Propagation, vol. 34, no. 3, pp. 276-280, 1986.

[7] R. Roy and T. Kailath, "ESPRIT-Estimation of signal parameters via rotational invariance techniques," IEEE Transactions on Acoustics, Speech, and Signal Processing, vol. 37, no. 7, pp. 984995, 1989.

[8] D. Malioutov, M. Çetin, and A. S. Willsky, "A sparse signal reconstruction perspective for source localization with sensor arrays," IEEE Transactions on Signal Processing, vol. 53, no. 8, pp. 3010-3022, 2005.

[9] M. M. Hyder and K. Mahata, "Direction-of-arrival estimation using a mixed $l_{2,0}$ norm approximation," IEEE Transactions on Signal Processing, vol. 58, no. 9, pp. 4646-4655, 2010.

[10] P. Stoica, P. Babu, and J. Li, "SPICE: a sparse covariance-based estimation method for array processing," IEEE Transactions on Signal Processing, vol. 59, no. 2, pp. 629-638, 2011.

[11] N. Nu, Z. Ye, and M. Bao, "DOA estimation for sparse array via sparse signal reconstruction," IEEE Transactions on Aerospace and Electronic Systems, vol. 49, no. 2, pp. 760-773, 2013.

[12] Z. M. Liu, Z. T. Huang, and Y. Y. Zhou, "Array signal processing via sparsity-inducing representation of the array covariance matrix," IEEE Transactions on Aerospace and Electronic Systems, vol. 49, no. 3, pp. 1710-1724, 2013.

[13] S. Yin, G. Wang, and H. Karimi, "Data-driven design of robust fault detection system for wind turbines," Mechatronics, 2013.

[14] A. C. Gurbuz, V. Cevher, and J. H. McClellan, "Bearing estimation via spatial sparsity using compressive sensing," IEEE Transactions on Aerospace and Electronic Systems, vol. 48, no. 2, pp. 1358-1369, 2012.

[15] E. T. Northardt, I. Bilik, and Y. I. Abramovich, "Spatial compressive sensing for direction-of-arrival estimation with bias mitigation via expected likelihood," IEEE Transaction on Signal Processing, vol. 61, no. 5, pp. 1183-1195, 2013.

[16] M. Carlin, P. Rocca, G. Oliveri, F. Viani, and A. Massa, "Direction of arrival estimation through Bayesian compressive sensing strategies," IEEE Transactions on Antennas and Propagation, vol. 61, no. 7, pp. 3828-3838, 2013.

[17] Z. Ye and C. Liu, "On the resiliency of MUSIC direction finding against antenna sensor coupling," IEEE Transactions on Antennas and Propagation, vol. 56, no. 2, pp. 371-380, 2008.

[18] L. Josefsson and P. Persson, Conformal Array Antenna Theory and Design, IEEE Press, Piscataway, NJ, USA, 2006.

[19] W. T. Li, X. W. Shi, Y. Q. Hei, S. F. Liu, and J. Zhu, "A hybrid optimization algorithm and its application for conformal array pattern synthesis," IEEE Transactions on Antennas and Propagation, vol. 58, no. 10, pp. 3401-3406, 2010.

[20] L. Zou, J. Lasenby, and Z. He, "Direction and polarization estimation using polarized cylindrical conformal arrays," IET Signal Processing, vol. 6, no. 5, pp. 395-403, 2012.

[21] Z.-S. Qi, Y. Guo, and B.-H. Wang, "Blind direction-of-arrival estimation algorithm for conformal array antenna with respect to polarisation diversity," IET Microwaves, Antennas and Propagation, vol. 5, no. 4, pp. 433-442, 2011.

[22] P. Yang, F. Yang, and Z. Nie, "DOA estimation using MUSIC algorithm on a cylinderical conformal array," in Proceedings of the IEEE Antennas and Propagation Society International Symposium, pp. 5299-5302, June 2007.

[23] P. Yang, F. Yang, and Z. P. Nie, "DOA estimation with sub-array divided technique and interporlated esprit algorithm on a cylindrical conformal array antenna," Progress in Electromagnetics Research, vol. 103, pp. 201-216, 2010.

[24] L. Wan, W. Si, L. Liu, Z. Tian, and N. Feng, "High accuracy 2D-DOA estimation for conformal array using PARAFAC," International Journal of Antennas and Propagation, vol. 2014, Article ID 394707, 14 pages, 2014.

[25] L. Wan, W. Si, L. Liu, and Z. Tian, "Joint estimation of 2DDOA and frequency based on space-time matrix and conformal array," The Scientific World Journal, vol. 2013, Article ID 463828, 10 pages, 2013.

[26] W. J. Si, L. T. Wan, L. T. Liu, and Z. X. Tian, "Fast estimation of frequency and 2-D DOAs for cylindrical conformal array antenna using state-space and propagator method," Progress in Electromagnic Research, vol. 137, pp. 51-71, 2013.

[27] L. Liu, Y. Jiang, L. Wan, and Z. X. Tian, "Beamforming of joint polarization-space matched filtering for conformal array," The Scientific World Journal, vol. 2013, Article ID 589675, 10 pages, 2013.

[28] S. Yin, S. Ding, A. Haghani, and H. Hao, "Data-driven monitoring for stochastic systems and its application on batch process," International Journal of Systems Science, vol. 44, no. 7, pp. 13661376, 2013.

[29] S. Yin, X. Yang, and H. Karimi, "Data-driven adaptive observer for fault diagnosis," Mathematical Problems in Engineering, vol. 2012, Article ID 832836, 21 pages, 2012.

[30] B.-H. Wang, Y. Guo, Y.-L. Wang, and Y.-Z. Lin, "Frequencyinvariant pattern synthesis of conformal array antenna with low cross-polarisation," IET Microwaves, Antennas and Propagation, vol. 2, no. 5, pp. 442-450, 2008. 


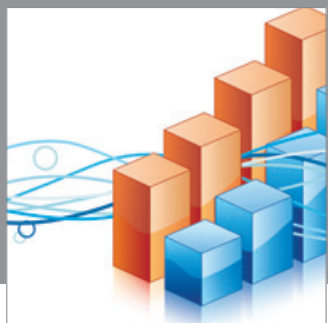

Advances in

Operations Research

mansans

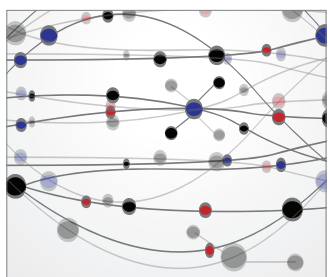

The Scientific World Journal
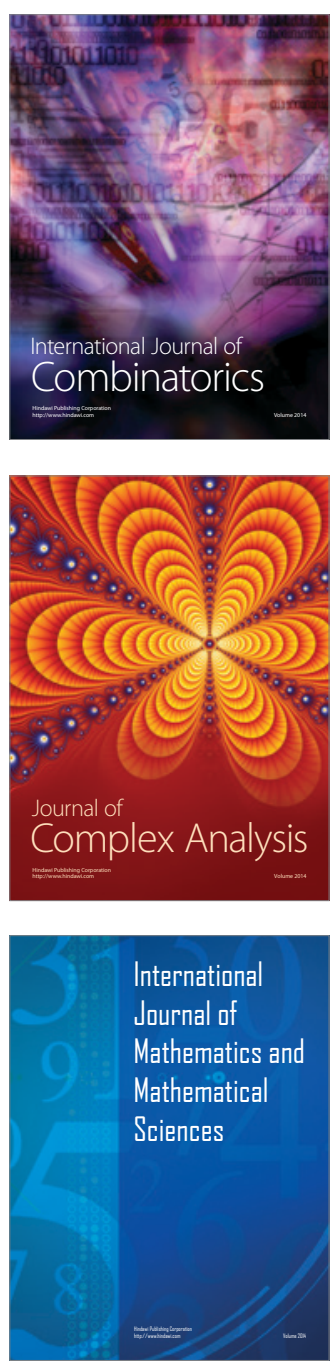
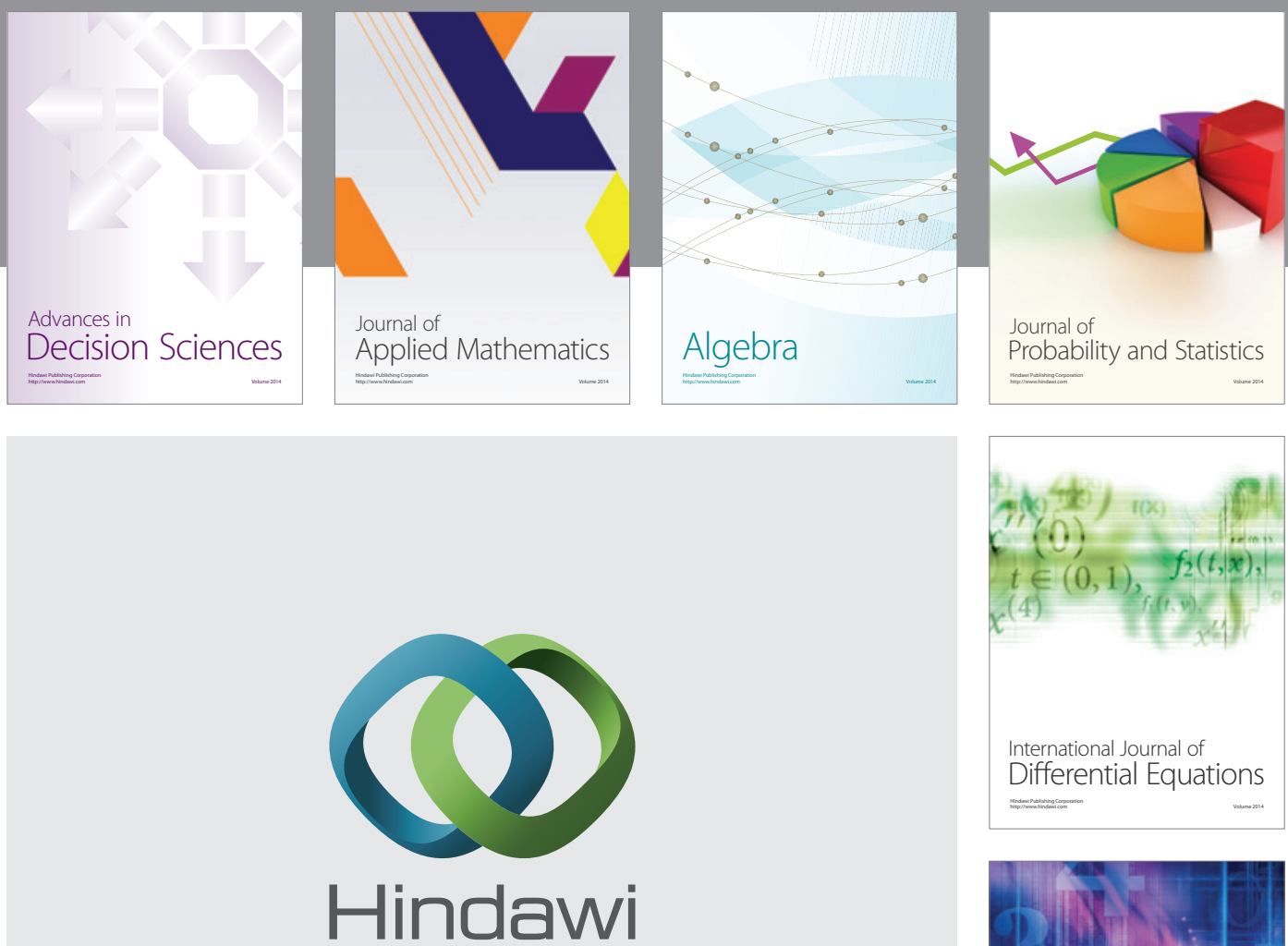

Submit your manuscripts at http://www.hindawi.com
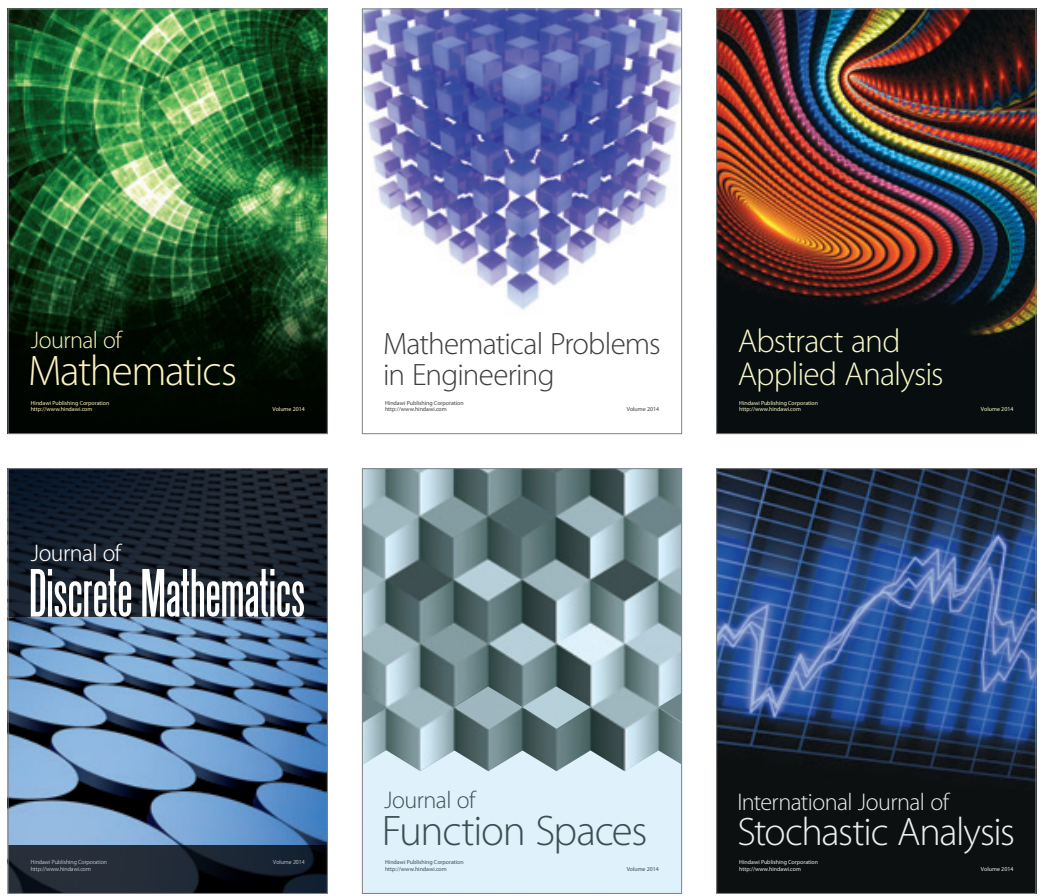

Journal of

Function Spaces

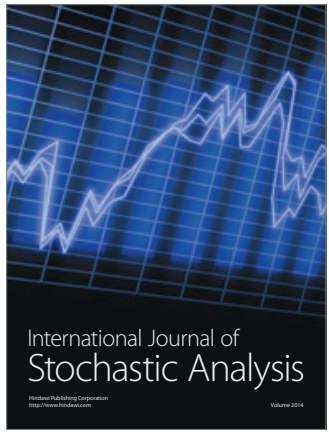

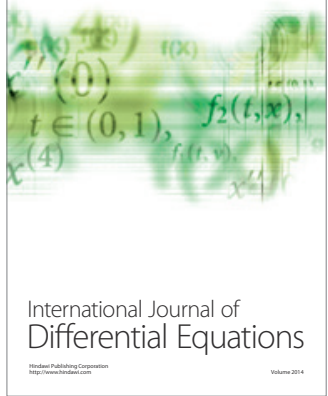
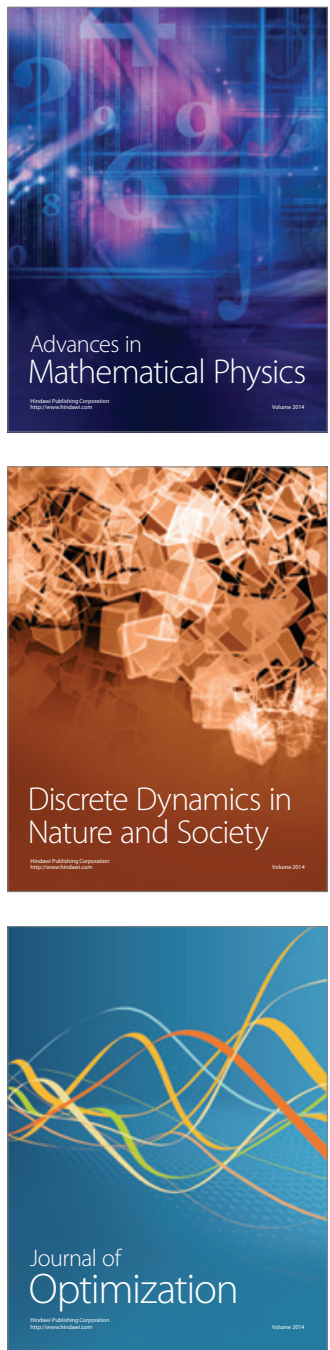